\title{
Anagrelide potentially provokes acute coronary syndrome even in an adolescent affected with essential thrombocythemia concomitant with underlying persistent coronary endothelial dysfunction
}

\author{
Ryuhei Tanaka ${ }^{1}$, Yodo Gotate ${ }^{1}$, Yasumori Sujino ${ }^{1}$, Kenji Fukushima ${ }^{1}$, Maki Todo ${ }^{1}$, \\ Atsuhiko Ohta ${ }^{1}$, Yuki Shimizu ${ }^{1}$, Atsuko Watanabe ${ }^{1}$, Ken Naganuma ${ }^{1}$, Toshihiro \\ Muramatsu $^{1}$, and Shintaro Nakano ${ }^{1}$ \\ ${ }^{1}$ Saitama Medical University International Medical Center
}

May 11, 2020

\begin{abstract}
Thrombohemorrhagic disorders are the main cause of morbidities and mortalities of essential thrombocythemia (ET), which are typically observed at age 50-60 years and rarely encountered in adolescence or childhood. Recently, anagrelide, a quinazinolone derivative, has been used as a therapeutic agent for ET. Although it is used to reduce platelet count, its cardiotoxicity has been reported. Here, we present an 18-year-old boy with ET who was treated with anagrelide and developed acute myocardial infarction. This was presumed to be an effect of anagrelide administration and, specifically, damage to the coronary arterial endothelial cell exacerbated by ET.
\end{abstract}

\section{Introduction:}

Essential thrombocythemia (ET) is a form of myeloproliferative neoplasms characterized by markedly increased mature megakaryocytes in the bone marrow and sustained thrombocytosis of peripheral blood. ${ }^{1,2}$ Patients with ET, especially those with mutations in the gene encoding Janus kinase 2 (JAK2 ), may experience thrombohemorrhagic complications due to multifactorial etiologies. ${ }^{3-6}$ Anagrelide is a non-leukemogenic cytoreductive drug used for the treatment of ET in patients with a long life-expectancy; however, it is associated with the incidence of various cardiovascular side effects (5\%-30\%). ${ }^{7-9}$ Here, we present an 18-year-old boy with ET who developed ST-segment elevation myocardial infarction (STEMI) occurring after anagrelide initiation.

\section{Case description:}

An 11-year-old boy was referred to the pediatric hematology/oncology department of our institution for investigation of increased platelet count $\left(1273 \times 10^{9} / \mathrm{L}\right)$. ET was diagnosed based on the hematological criteria including abnormal bone marrow findings (Fig. 1A), identification of the JAK2 V617F mutation, and absence of the $B C R$ - $A B L 1$ fusion gene. ${ }^{1,2}$ His mother reported that he had intractable headache since age 2 . The migraine-like severe headache was determined to be a vasomotor symptom of ET. ${ }^{10}$ Low-dose aspirin (100 $\mathrm{mg}$ /day) was administered to attenuate the microcirculatory symptom, which successfully ameliorated his headache. ${ }^{2,}{ }^{10}$ However, the platelet count remained high for years, reaching $1250 \times 10^{9} / \mathrm{L}$ by the age of 18 . Eventually, an intramuscular hemorrhage developed in the right buttock. Based on current epidemiological evidence, he was assumed to be at a high-risk of thrombohemorrhagic events. ${ }^{2,}{ }^{8}, 11$; therefore, cytoreductive therapy was started. Because of the concerns of the leukemogenic potential, we administered anagrelide (1.0 $\mathrm{mg}$ /day) instead of hydroxyurea. ${ }^{12,13}$ We confirmed that cardiac function at baseline was normal to initiate drug therapy because of its toxic potential. ${ }^{7-9}$ Anagrelide successfully reduced the platelet count (range, 
500-700 $\times 10^{9} / \mathrm{L}$ ). Clinically, there was no recurrence of thrombohemorrhagic events; however, he periodically complained of palpitations or vague chest sensations.

Five months after the anagrelide initiation, he was transferred to our cardiac center because of progressive worsening of precordial discomfort. On arrival, he was conscious with a body temperature, $36.5^{\circ} \mathrm{C}$; heart rate, 87 beats/min; and systolic/diastolic pressure, $133 / 87 \mathrm{mmHg}$. The electrocardiogram showed sinus rhythm and prominent ST-T elevation in leads II, III, aVF, and V1-V3 with a reciprocal change (Fig. 1B). Blood tests revealed leukocytosis (white blood cell count of $14.7 \times 10^{9} / \mathrm{L}$ with neutrophils $81.5 \%$ ), thrombocytosis (platelet count $894 \times 10^{9} / \mathrm{L}$ ), and elevated markers for cardiac injury (troponin I $1288 \mathrm{pg} / \mathrm{mL}$, creatine-kinase $9734 \mathrm{IU} / \mathrm{L})$. He had no smoking history. Emergent coronary angiography revealed total middle right coronary artery (RCA) occlusion and spastic stenosis of the distal left anterior descending artery (LAD) (Figs. 1C and 1D). The RCA was recanalized by thrombus aspiration and intra-coronary nitroglycerin infusion, whereas the LAD was dilated with the intra-coronary nitroglycerin infusion alone (Figs. $1 \mathrm{E}$ and $1 \mathrm{~F}$ ). ${ }^{99 \mathrm{~m}}$ Technetiumpyrophosphate scintigraphy was performed 3 days later; cardiac magnetic resonance imaging performed 10 days later demonstrated extensive myocardial infarction in the broad inferior and apical anterior wall (Figs. $2 \mathrm{~A}-\mathrm{C})$.

Anagrelide was considered as a potential causative agent for the STEMI; thus, its administration was ceased. Instead, hydroxyurea $(1000 \mathrm{mg} /$ day $)$ together with low-dose aspirin $(100 \mathrm{mg} /$ day $)$ was initiated, which successfully controlled the platelet count (platelet count 500-700 $\times 10^{9} / \mathrm{L}$ ) without any recurrence of thrombohemorrhagic events. To date, the patient has been asymptomatic regarding cardiovascular manifestations, with the continuation of a renin-angiotensin-aldosterone-system inhibitor (enalapril, $10 \mathrm{mg} /$ day) and coronary vasodilators (diltiazem, $200 \mathrm{mg} /$ day; isosorbide, $40 \mathrm{mg} /$ day).

Follow-up coronary angiography with the acetylcholine provocation test was performed 12 months later after a 2-day interruption of these vasodilators. Severe multivessel vasospasm was induced by intra-coronary acetylcholine infusion (Figs. 2D-G). Optical coherence tomography of the RCA (Fig. 2H) suggested intimal fibrotic changes without a remarkable atheromatous plaque; thus, continuous administration of the coronary vasodilators was required to prevent coronary ischemia recurrence.

\section{Discussion}

Relatively young patients (age 30-45) with ET who do not exhibit traditional coronary risk factors (i.e., dyslipidemia, hypertension, smoking, diabetes, and older age) have also been reported to have acute myocardial infarction $(\mathrm{AMI})^{14-17}$; however, there is little information on the incidence of AMI among patients younger than 20 years, with few reports on such cases. ${ }^{18}$

The mechanisms underlying acute coronary syndrome are considered to be multifactorial. Increased blood cells numbers and increased blood viscosity in ET promote the formation of platelet-leukocyte aggregates, with concomitant release of proteases from the activated leukocytes into circulation, and consequent intravascular hypercoagulability. ${ }^{19}$ Besides the hyperviscosity, chronic endothelial cell damage provoked by activated platelets and chronic high shear stress on the vessel wall are potential causative factors for acute coronary syndrome. ${ }^{19}$ Most patients with ET (approximately 60\%) harbor the JAK2 V617F mutation in their hematopoietic cells. ${ }^{2}{ }^{20}$ This somatic mutation causes constitutive activation of the kinase, resulting in myeloproliferative disorders, and is also known as a significant risk factor for cardiovascular events. ${ }^{2-6,}{ }^{16}$ Pathophysiologically, platelets from patients with ET carrying the JAK2 V617F mutation increased their capacity to generate thrombin associated with platelet activation. ${ }^{3}$ Additionally, megakaryocytes from the JAK2 V617F knocked-in model mice showed accelerated maturation and increased proplatelet formation, ${ }^{21}$ indicating that the $J A K 2 \mathrm{~V} 617 \mathrm{~F}$ mutation alters the intrinsic characters of both megakaryocytes and platelets beyond merely increasing in cell numbers. In our case, coronary endothelial cell dysfunction lasted for a year after STEMI onset and was confirmed by a positive acetylcholine provocation test result. We suggest that the presence of the JAK2 V617F mutation and hyperviscosity since childhood, may have contributed to the persistent chronic coronary pathology.

Anagrelide is a selective inhibitor of thrombopoiesis and is effective for lowering the platelet count. ${ }^{9,22-24}$ 
Although the precise mechanism underlying the attenuation of platelet overproduction remains ambiguous, anagrelide's effect may be based on the repression of transcriptional factors related to the megakaryopoiesis including GATA-1 and FOG-1. ${ }^{25}$ Furthermore, anagrelide inhibits cyclic adenosine monophosphate phosphodiesterase III pharmacologically, and has inotropic and vasodilator properties; thus, the most common cardiovascular adverse events associated anagrelide include tachycardia/palpitations. ${ }^{9}, 12$ However, acute coronary syndrome including AMI and angina have been reported as adverse events of anagrelide. ${ }^{23,}{ }^{25} \mathrm{Ty}-$ pically, patients with ET are middle-aged or older ${ }^{2}$ and often have the traditional cardiovascular risk factors. Therefore, observation of AMI pathogenesis in our very young patient without any conventional risk factors, except for ET itself and anagrelide administration, was unusual. ${ }^{13}$

The biological effects of anagrelide on the coronary artery are controversial, and both vasospasm ${ }^{25}$ and vasodilation ${ }^{26}$ have been reported. Theoretically, the inhibition of phosphodiesterase III by anagrelide should induces vasodilatation, but not vasospasm. However, a mouse model study revealed that phosphodiesterase inhibitors increased the release of sympathetic neurotransmitters. ${ }^{27} \mathrm{~A}$ report speculated that depending on the expression patterns of alpha- and beta- adrenergic receptors in particular vessels cause vasoconstriction and vasodilatation, respectively. ${ }^{25}$ In addition to the long-lasting endothelial cell damage caused by ET itself, the complex distribution of adrenergic receptors may induce the deterioration of coronary vessel function, eventually resulting in STEMI. In our case, the negative effects of thrombocytosis due to ET on the maintenance of coronary artery integrity, as shown by abnormal findings, together with the pharmacological effects anagrelide on the artery led to the exacerbation of chest symptoms such as palpitations and vague sensations, which finally led to STEMI.

In conclusion, our patient with ET developed STEMI as a consequence of anagrelide administration and persistent coronary endothelial dysfunction caused by the ET itself.

$1200 / 1200$ words

\section{Conflict of interest:}

The authors declare that they have no conflict of interest.

\section{Acknowledgments:}

We would like to thank Editage for editing and reviewing this manuscript for English language.

\section{References}

1. Arber DA, Orazi A, Hasserjian R, et al. The 2016 revision to the World Health Organization classification of myeloid neoplasms and acute leukemia. Blood 2016; 127:2391-2405.

2. Tefferi A, Pardanani A. Essential thrombocythemia. N Engl J Med 2019; 381: 2135-2144.

3. Falanga A, Marchetti M. Thrombosis in myeloproliferative neoplasms. Semin Thromb Hemost 2014; 40: 348-358.

4. Etheridge SL, Roh ME, cosgrove ME, et al. JAK2V617F-positive endothelial cell contribute to clotting abnormalities in myeloproliferative neoplasms. Proc Natl Acad Sci USA 2014; 111: 2295-2300.

5. Guy A, Gourdou-Latyszenok V, Lay NL, et al. Vascular endothelial cell expression of JAK2V617F is sufficient to promote a pro-thrombotic state due to increased P-selectin expression. Haematologica 2019; 104: $70-81$

6. Poisson J, Tanguy M, Davy H, et al. Erythrocyte-derived microvesicles induce arterial spasm in JAK2V617F myeloproliferative neoplasm. J Clin Invest 2020; 130: 2630-2643.

7. Gugliotta L, Tieghi A, Tortorella G, et al. Low impact of cardiovascular adverse events on anagrelide treatment discontinuation in a cohort of 232 patients with essential thrombocythemia. Leuk Res 2011; 35: $1557-1563$. 
8. Tortorella G, Piccin A, Tieghi A, et al. Anagrelide treatment and cardiovascular monitoring in essential thrombocythemia. A prospective observational study. Leuk Res 2015; 39: 592-598.

9. Birgegard G, Besses C, Griesshammer M, et al. Treatment of essential thrombocythemia in Europe: a prospective long-term observational study of 3649 high-risk patients in the evaluation of anagrelide efficacy and long-term safety study. Haematologica 2018; 103: 51-60.

10. Griesshammer M, Bangerter M, Vliet H, Michiels JJ. Aspirin in essential thrombocythemia: Status Quo and Quo Vadis. Semin Thromb Hemost 1997; 23: 371-377.

11. De Stefano V, Za T, Rossi E, et al. Recurrent thrombosis in patients with polycythemia vera and essential thrombocythemia: incidence, risk factors, and effect of treatments. Haematologica 2008; 93: 372-380.

12. Mazzucconi MG, Redi R, Bernasconi S, et al. A long-term study of young patients with essential thrombocythemia treated with anagrelide. Haematologica 2004; 89: 1306-1313.

13. Fu R, Zhang L, Yang R. Paederiac essential thrombocythemia: clinical and molecular features, diagnosis and treatment. Br J Haematol 2013; 163; 295-302.

14. Bildirici U, Celikyurt U, Ural E. Essential thrombocythemia: a case of acute ST-segment elevation myocardial infarction in a young female. Clin Cardiol 2009; 32: 104-105.

15. Lim YH, Lee YY, Kim JH, et al. Development of acute myocardial infarction in a young female patient with essential thrombocythemia treated with anagrelide: a case report. Korean J Hematol 2010; 45: 136-138.

16. Lata K, Madiraju N, Levitt L. JAK2 mutations and coronary ischemia. N Engl J Med 2010; 363: 396-397.

17. Sibellas F, Schaaf M. Acute myocardial infarction and anagrelide. Int J Cardiol 2013; 168: e50-e52.

18. Rossi C, Randi ML, Zerbinati P, Rinaldi V, Girolami A. Acute coronary disease in essential thrombocythemia and polycythemia vera. J Inter Med 1998; 244: 49-53.

19. Cella G, Marchetti M, Vianello F, et al. Nitric oxide derivatives and soluble plasma selectins in patients with myeloproliferative neoplasmas. Thronb Haemost 2010; 104: 151-156.

20. Tefferi A, Guglielmelli P, Larson DR, et al. Long-term survival and blast transformation in molecularly annotated essential thrombocythemia, polycythemia vera, and myelofibrosis. Blood 2014; 124: 2507-2513.

21. Hobbs $\mathrm{CH}$, Manning H, Bennett $\mathrm{C}$, et al. JAK2V617F leads to intrinsic change in a knock-in mouse model of essential thrombocythemia. Blood 2013; 122: 3787-3797.

22. Harrison CN, Bareford D, Butt N, et al. Guideline for investigation and management of adults and children presenting with a thrombocytosis. Br J Haematol 2010; 149: 352-375.

23. Gisslinger H, Gotic M, Holowiecki J, et al. Anagrelide compared with hydroxyurea in WHO-classified essential thrombocythemia: the ANAHYDRET study, a randomized controlled trial. Blood 2013; 121: 17201728 .

24. Harrison CN, Campbell PJ, Buck G, et al. Hydroxyurea compared with anagrelide in high-risk essential thrombocythemia. N Engl J Med 2005; 353: 33-45.

25. Ahluwalia M, Donovan H, Singh N, Butcher L, Erusalimski D. Anagrelide represses GATA-1 and FOG-1 expression without interfering with thrombopoietin receptor signal transduction. J Thromb Haemost 2010; 8: $2252-2261$.

26. Lin GM, Chao TY, Wang WB. Acute coronary syndromes and anagrelide. Int J Cardiol 2007; 117: e17-e19

27. Piccin A, Steurer M, Feistritzer C, et al. Observational retrospective study of vascular modulator changes during treatment in essential thrombocythemia. Transl Res: 2017; 184: 21-34. 
28. Johnston H, Majewski H, Musgrave IF. Involvement of cyclic nucleotides in prejunctional modulation of noradrenaline release in mouse atria. Br J Pharmacol 1987; 91: 773-781.

\section{Figure legends:}

\section{Figure.1}

Representative microscopic image (A) of the bone marrow sample stained with hematoxylin and eosin; (B) electrocardiogram showing sinus rhythm and prominent ST-T elevation in leads II, III, aVF, and V1-V3 (arrowheads) with a reciprocal change; (C, E) coronary angiograms showing occlusion of the right coronary artery which was recanalized through thrombus aspiration and intra-coronary nitroglycerin infusion (arrowheads); (D, F) spastic left anterior descending artery, which was dilated only with the intra-coronary nitroglycerin infusion (arrows). Abbreviations: NTG, nitroglycerin.

\section{Figure 2}

Representative images from PYP and CMR; (A) PYP showed significant uptake in the inferior and apical walls including the right ventricle (yellow arrows); T2-weighted CMR image showing (B) high signal intensity in the inferoseptal wall and right ventricle (arrows); delayed enhancement was observed in the area with myocardial infarction $(\mathrm{C})$ (arrow heads).

Representative images from follow-up coronary angiography with acetylcholine provocation test and OCT of the RCA; coronary angiogram (arrow heads indicate the right coronary artery; arrows indicate the left anterior descending artery) showing (D and $\mathrm{E}$ ) non-occlusive coronary arteries at baseline and ( $\mathrm{F}$ and $\mathrm{G}$ ) total occlusion upon intra-coronary acetylcholine infusion; (H) OCT of the RCA showing extensive, homogenous bright intimal thickening (yellow arrows) without prominent attenuation, suggesting fibrotic change.

Abbreviations: Ach, acetylcholine; OCT, optical coherence tomography; RCA, right coronary artery; PYP, ${ }^{99 \mathrm{~m}} \mathrm{Tc}$-pyophosphate scintigraphy; CMR, cardiac magnetic resonance imaging.

\section{Figure 1}
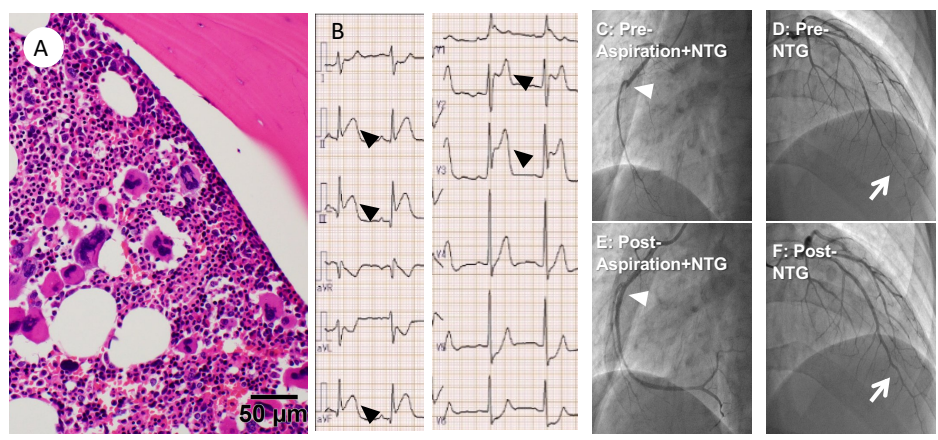
Figure 2

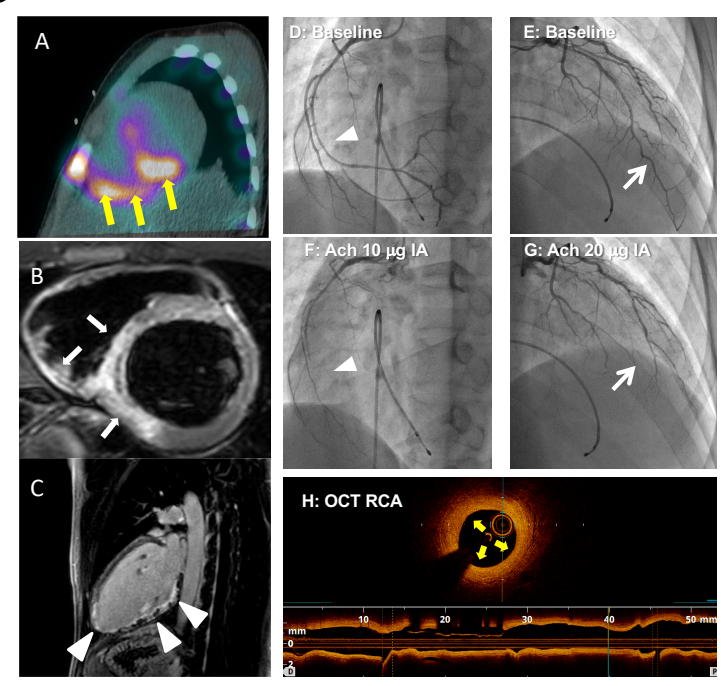

